\title{
SENSIBILIDADE DE Aspergillus sp. E Fusarium sp. A DIFERENTES CONCENTRAÇÕES DE FUNGICIDAS
}

\author{
José Roberto Chaves Neto ${ }^{1}$, Ricardo Boscaini ${ }^{2}$, Ivan Francisco Dressler da Costa $^{3}$
}

\section{RESUMO:}

A principal estratégia de controle das doenças relacionadas aos grãos e espigas na cultura do milho é a aplicação de fungicidas. No entanto, ao longo dos últimos anos vem ocorrendo o desenvolvimento de maior resistência dos fungos perante as moléculas químicas. Com isso, a determinação in vitro de espécies resistentes a estes produtos possibilita o desenvolvimento de novas formas de controle. O objetivo deste trabalho foi avaliar a sensibilidade in vitro dos fungos Aspergillus sp. e Fusarium sp. a diferentes fungicidas. Foram avaliados os fungicidas Epoxiconazol + Piraclostrobina, Ciproconazol + Picoxistrobina e Fluxapiroxade + Piraclostrobinal nas concentrações de 0,10, 50, 100, 500, 1000 ppm, sob delineamento inteiramente casualizado, em arranjo fatorial, com quatro repetições. Determinou-se a porcentagem de inibição de crescimento micelial, o índice de velocidade do crescimento micelial e a concentração de cada fungicida capaz de inibir $50 \%$ do crescimento micelial ( $\mathrm{IC}_{50}$ ). De modo geral, quanto maior a concentração do fungicida utilizado maior foi a porcentagem de inibição e menor índice de velocidade média de crescimento micelial dos fungos Aspergillus sp. e Fusarium sp. Para o fungo Aspergillus sp., o fungicida Fluxapiroxade + Piraclostrobina proporcionou o menor crescimento micelial com a $\mathrm{IC}_{50}$ de 39,78 ppm. Já para o fungo Fusarium sp., o menor crescimento micelial foi observado com o fungicida Epoxiconazol + Piraclostrobina na $\mathrm{IC}_{50}$ de $25,56 \mathrm{ppm}$.

Palavras-chave: controle químico, estrobilurinas, triazóis

\section{SENSITIVITY OF Aspergillus sp. And Fusarium sp. TO DIFFERENT CONCENTRATIONS OF FUNGICIDES}

\begin{abstract}
:
The application of fungicides is the main strategy to control diseases related to grains and corn on the cob crop. However, over the last few years there has been noticed a development of greater resistance of fungi to chemical molecules. This has allowed for the development of new forms of control, when the species resistant to this product are determined in vitro. The objective of this study was to evaluate the in vitro sensitivity of the fungi Aspergillus sp. and Fusarium sp. to different fungicides. The fungicides Epoxiconazole + Piraclostrobin, Ciproconazol + Picoxystrobin and Fluxapiroxade + Piraclostrobinal were evaluated at concentrations of $0,10,50,100,500,1000 \mathrm{ppm}$, under a completely randomized design, in a factorial arrangement, with four replications. The percentage of mycelial growth inhibition, the rate of mycelial growth rate and the concentration of each fungicide capable of inhibiting $50 \%$ of mycelial growth
\end{abstract}

\footnotetext{
${ }^{1}$ Eng. Agrônomo, Doutor em Engenharia Agrícola (PPGEA/UFSM), Santa Maria, RS. jose.chavesneto@ gmail.com;

${ }^{2}$ Eng. Agrônomo, Doutorando em Engenharia Agrícola (PPGEA/UFSM), Santa Maria, RS. ricardoboscaini75@gmail.com

${ }^{3}$ Eng. Agrônomo, Doutor em Agronomia (UFSM), Santa Maria, RS. ifdresler@gmail.com
} 
Revista Agri-Environmental Sciences, Palmas-TO, v. 6, e020003, 2020

ISSN 2525-4804

(IC50) were determined. In general, the higher the concentration of the fungicide used, the greater the percentage of inhibition and the lowest average rate of mycelial growth of the fungi Aspergillus sp. and Fusarium sp. For the fungus Aspergillus sp., the fungicide Fluxapiroxade + Piraclostrobina provided the lowest mycelial growth with the IC50 of 39.78 ppm. For the fungus Fusarium sp., the lowest mycelial growth was observed with the fungicide Epoxiconazole + Pyraclostrobin in the IC50 of $25.56 \mathrm{ppm}$. sp.

Keywords: Chemical control, strobilurins, triazoles. 


\section{INTRODUÇÃO}

Algumas espécies de fungos dos gêneros Penicillium sp., Aspergillus sp. e Fusarium sp., podem causar, em condições de campo, podridão da espiga e produção de grãos de milho (Zea mays L.) ardidos, já que consomem as reservas armazenadas pela fotossíntese, além de serem capazes de biossintetizar em condições de armazenamento micotoxinas (Mendes et al., 2011). Essas toxinas provocam toxicidade aguda em diversos animais, especialmente aves suínos e equinos, podendo leválos à morte (Oliveira et al., 2015).

As micotoxinas são compostos tóxicos produzidos por fungos. Elas são altamente nocivas à saúde animal e humana e ocasionam grandes perdas econômicas. Dentre elas destacam-se as aflatoxinas B1, B2, G1 e G2, que são biosintetizadas por fungos predominantemente do gênero Aspergillus, como A. flavus, A. parasiticus, A. nomius. Já a zearalenona são produzidas por várias espécies de Fusarium, com ocorrência em milho, cevada (Hordeum vulgare), trigo (Triticum spp.) e aveia (Avena sativa). As fumonisinas são produzidas por espécies do gênero Fusarium, mais exatamente $F$. verticillioides. A zearalenona é outro tipo de micotoxina biosintetizada por Fusarium, como $F$. graminearum, $F$. culmorum, $F$. equiseti e $F$. cerealis, que contaminam cereais, incluindo o milho e diversas frutas como banana (Musa spp.) e tomate (Solanum lycopersicum) (Rufatto, 2011; Vitorino, 2011).

A aplicação foliar de fungicidas é o principal método de controle das podridões de grãos e espiga na cultura do milho. A eficácia do controle químico está relacionada tanto a facilidade de aplicação quanto aos resultados rápidos, o que justifica a grande utilização dos fungicidas por parte dos produtores nas últimas décadas (Ghini e Kimati, 2009; Reis et al., 2010; Lopes et al., 2015). Os fungicidas sistêmicos pertencentes aos grupos químicos dos triazóis são os mais empregados por inibirem a biossíntese de esterol na célula fúngica, e sua maior eficácia é observada nas fases de colonização e na pré-esporulação dos patógenos (Zhou e Wang, 2012; Silva et al., 2013). Outros fungicidas utilizados são os formulados a partir das estrobirulinas isoladas ou em misturas pré-fabricadas responsáveis pela inibição da respiração mitocondrial, apresentando maior efeito na fase de germinação dos esporos (Bampi et al., 2013; Frac, 2014).

Porém, nos últimos anos vem sendo observado maior resistência dos fungos fitopatogênicos perante a determinadas moléculas químicas (Hawkins et al., 2018). A resistência relativa dos fungos ao fungicida é um fenômeno conhecido e relatado em muitas regiões produtoras. Esta resistência é proporcional à especificidade do modo de ação do fungicida, sendo os fungicidas sistêmicos considerados como mais específicos e seletivos e que apresentam maior predisposição a resistência. (Reis et al., 2010; Bolton et al., 2012; Souza et al., 2012).

A fungitoxicidade de um determinado fungicida caracteriza-se pela toxicidade de determinada substância presente em baixas concentrações de fungos. Sua função consiste na redução ou inibição do desenvolvimento de microrganismos, pois sua capacidade é tida como uma propriedade inerente a determinada substância química (Edgington et al., 1971; Reis et al., 2010). Diferentes critérios têm sido utilizados para avaliar a fungitoxicidade de uma substância química. A exemplo pode-se utilizar os parâmetros de $\mathrm{DE}_{50}$ ou $\mathrm{IC}_{50}$ (dose efetiva), DL50 (dose letal), $\mathrm{CL}_{50}$ (concentração letal), $\mathrm{CE}_{50}$ (concentração efetiva), ou CMI (concentração mínima inibitória), que inibe $50 \%$ do crescimento micelial ou da germinação de esporos (Edgington et al., 1971; Pfeufer e Ngugi, 2012).

Um fungo pode apresentar ou não sensibilidade à determinada substância química. Esta sensibilidade pode estar relacionada a fatores genéticos do patógeno, a fatores climáticos e até mesmo à eficiência da aplicação. Quando o fungo é sensível a um determinado fungicida, considera-se o fungicida tóxico; porém, quando não constatada a 
sensibilidade, este é determinado como não tóxico. Da mesma forma, se o fungicida não mostra fungitoxicidade sobre o fungo, este é considerado insensível ou resistente (Garcia e Juliatti, 2012; Tonin et al., 2013; Santos et al., 2017).

Desse modo, o monitoramento da sensibilidade de uma população de fungos alvos é essencial para determinar a presença de isolados resistentes. Pesquisadores têm se referido ao fenômeno da resistência como uma perda de sensibilidade dos fungos aos produtos, resultando em uma diminuição da eficiência destes sob condições de campo (Ghini e Kimati, 2009; Santos et al., 2017).

Diante do exposto, o presente trabalho teve como objetivo avaliar a sensibilidade in vitro dos fungos Aspergillus sp. e Fusarium sp. a diferentes fungicidas sobre o crescimento micelial, à inibição do crescimento micelial, ao índice de velocidade do crescimento micelial e à determinação os valores de $\mathrm{IC}_{50}$.

\section{MATERIAL E MÉTODOS}

Os experimentos foram conduzidos na Clínica Fitossanitária do Departamento de Defesa Fitossanitária do Centro de Ciências Rurais da Universidade Federal de Santa Maria, Santa Maria, RS, no período de julho a agosto de 2015.

Para ambos os fungos estudados, o delineamento experimental utilizado foi $o$ inteiramente casualizado, em arranjo fatorial, constituído por três fungicidas e seis concentrações $(0,10,50,100,500$ e 1000 ppm). O tratamento zero constou como testemunha e consistiu de placas de Petri com BDA (batata-dextrose-ágar) sem fungicida, com quatro repetições (Tabela 1).

Foram utilizados isolados de fungos Aspergillus sp. e Fusarium sp. associados a grãos de milho naturalmente infectados. Foram distribuídos 200 grãos de milho no interior de oito caixas tipo gerbox plástico, os quais foram armazenados em câmara de crescimento sob iluminação contínua, à temperatura de $25 \pm 2{ }^{\circ} \mathrm{C}$, por um período de incubação de nove dias. Após esse período, os fungos foram identificados em nível de gênero com o auxílio de microscópio ótico e seus esporos foram transferidos para placas de Petri contendo BDA com auxílio de uma agulha histológica, sendo cultivados por sete dias à temperatura de $25 \pm 2{ }^{\circ} \mathrm{C}$ e fotoperíodo de $12 \mathrm{~h}$. Após esse período, um disco de micélio foi transferido para novas placas de Petri com BDA para a obtenção de culturas puras de cada isolado, sendo incubadas sob as condições descritas acima.

Tabela 1. Ingrediente ativo (i.a.), produto comercial (p.c.) e concentração (g i.a $\mathrm{L}^{-1} \mathrm{ou} \mathrm{Kg}^{-1}$ do produto comercial) utilizadas no ensaio in vitro para os fungos Aspergillus sp. e Fusarium sp.

\begin{tabular}{ccc}
\hline Tratamentos (i.a) & $\begin{array}{c}\text { Produto } \\
\text { Comercial }\end{array}$ & $\begin{array}{c}\text { Concentração } \\
\left(\mathbf{g ~ L}^{-\mathbf{1}}\right)\end{array}$ \\
\hline Testemunha & --- & -- \\
\hline $\begin{array}{c}\text { Epoxiconazol + } \\
\text { Piraclostrobina }\end{array}$ & Abacus HC $^{\circledR}$ & $(333+167)$ \\
\hline $\begin{array}{c}\text { Ciproconazol + } \\
\text { Picoxistrobina }\end{array}$ & $\begin{array}{c}\text { Aproach } \\
\text { Prima }^{\circledR}\end{array}$ & $(80+200)$ \\
\hline $\begin{array}{c}\text { Fluxapiroxade }+ \\
\text { Piraclostrobina }\end{array}$ & Orkestra SC $^{\circledR}$ & $(167+333)$ \\
\hline
\end{tabular}

Após a obtenção das culturas puras, preparou-se uma solução estoque (SE) de 1000 ppm de cada tipo de fungicida, das quais foram obtidas as concentrações de 10, 50, 100 e 500 ppm. Posteriormente, transferiu-se uma alíquota $(2 \mathrm{~mL}) \mathrm{de}$ cada uma das concentrações dos diferentes fungicidas ao meio BDA fundido, com temperatura média de $38^{\circ} \mathrm{C}$. Em seguida, $20 \mathrm{~mL}$ do meio BDA contendo as diferentes concentrações de cada um dos fungicidas foi vertido em placas de Petri de nove $\mathrm{cm}$ de diâmetro, em câmara de fluxo laminar (Lopes et al., 2015). Após a solidificação do meio de cultura nas placas de Petri, discos de micélios $(5 \mathrm{~mm})$ de cada um dos isolados, separadamente, foram transferidos para as placas, as quais foram incubadas por 10 dias, sob temperatura de $25^{\circ} \pm 2{ }^{\circ} \mathrm{C}$ e fotoperíodo de $12 \mathrm{~h}$. 
As avaliações foram realizadas por meio de medições diárias do diâmetro das colônias, com auxílio de um paquímetro digital, em eixos ortogonal (média das duas medidas diametralmente opostas) para obter-se a média do crescimento micelial diário, até que o tratamento testemunha atingisse os bordos da placa (Maia et al., 2011). A partir dos dados obtidos, calculou-se a porcentagem de inibição de crescimento micelial (PICM) e o índice de velocidade do crescimento micelial (IVCM).

A PICM foi determinada através da equação 1, citada por Garcia et al. (2013):

$$
\operatorname{PICM}(\%)=\frac{(\mathrm{DTT}-\mathrm{DTQ})}{\mathrm{DTT}} \times 100 \quad(\text { Equação } 1)
$$

Em que: DTT = diâmetro no tratamento testemunha $(\mathrm{mm})$; DTQ $=$ diâmetro no tratamento químico $(\mathrm{mm})$.

O IVCM foi determinado com base na equação 2, descrita Maia et al. (2011):

$$
=\sum \frac{(\mathrm{D}-\mathrm{Da})}{\mathrm{IVCM}(\mathrm{mm} \text { dia }-1)}
$$

Em que: $\mathrm{D}$ = diâmetro médio atual da colônia $(\mathrm{mm}) ; \mathrm{Da}=$ diâmetro médio da colônia do dia anterior (mm); $\mathrm{N}=$ número de horas ou dias após a inoculação.

Para determinar a concentração de cada fungicida capaz de inibir $50 \%$ do crescimento micelial $\left(\mathrm{IC}_{50}\right)$, gerou-se, inicialmente, a equação de regressão, a partir da qual foi estimada a concentração de cada fungicida capaz de inibir $50 \%$ do crescimento micelial $\left(\mathrm{IC}_{50}\right)$. Após a obtenção da $\mathrm{IC}_{50}$ realizou-se a classificação dos diferentes fungicidas quanto a sensibilidade dos fungos, com base nos critérios e classes propostas por Edgington et al. (1971) ao definirem que a fungitoxicidade de uma determinada substância fungicida concentra os seguintes índices: $\mathrm{IC}_{50}<1 \mathrm{ppm}=$ altamente fungitóxico, $\mathrm{IC}_{50}$ de $1-50 \mathrm{ppm}=$ moderadamente fungitóxica e $\mathrm{IC}_{50}>50 \mathrm{ppm}=$ não tóxico.

Os dados obtidos formam submetidos aos testes de normalidade e homogeneidade dos erros, e constatou-se a não necessidade de transformá-los. Em seguida, os dados foram submetidos à análise de variância. Quando observado a significância, as variáveis qualitativas tiveram suas médias comparadas pelo teste de Tukey, ao nível de 5\% de probabilidade. Já as médias das variáveis quantitativas foram submetidas a uma análise de regressão com auxílio do programa estatístico Sisvar versão 5.6 (Ferreira, 2014).

\section{RESULTADOS E DISCUSSÃO}

Os resultados da análise da variância e dos valores médios de crescimento micelial, PICM e IVCM dos isolados de Aspergillus sp. e Fusarium sp., indicam que existe diferença significativa entre os tratamentos de cada fator, assim como na interação entre os fatores de variação: Concentração, Fungicidas, Concentração x Fungicidas (Tabela 2). Os baixos valores de coeficiente de variação mostram que os dados observados satisfazem a premissa de normalidade.

Ao analisar os diferentes tipos de fungicidas utilizados, constatou-se diferença siginificativa $(\mathrm{p} \leq 0,05)$ para a porcentagem de inibição do crescimento micelial dos fungos Aspergillus sp. e Fusarium sp (Tabela 3).

Para o fungo Aspergillus sp., constou-se que o fungicida Fluxapiroxade + Piraclostrobina foi o que obteve a maior porcentagem de inibição do crescimento micelial, com média superior a $60 \%$. O fungicida que proporcionou a menor porcentagem de inibição foi o Ciproconazol + Picoxistrobina, com média de $10 \%$ (Tabela 3).

Estes resultados mostram a eficácia dos princípios ativos pertencentes ao grupo químico das estrobilurinas. Esta eficácia também foi reportada por Chaves Neto et al. (2017), visto que obtiveram 
como resultado eficácia superior a $90 \%$ no controle de mancha branca do milho em condições de campo, para os fungicidas Epoxiconazol + Piraclostrobina,
Ciproconazol + Picoxistrobina e Azoxistrobina + Benzovindiflupir, ambos com um dos princípios ativos do grupo das estrobilurinas.

Tabela 2. Resumo da análise de variância e valores médios de crescimento micelial (CM), porcentagem de inibição de crescimento micelial (PICM) e índice de velocidade do crescimento micelial (IVCM) dos fungos Aspergillus sp. e Fusarium sp. isolados de grãos de milho.

\begin{tabular}{|c|c|c|c|c|c|c|c|}
\hline \multirow{3}{*}{${ }^{1} \mathbf{F V}$} & \multirow{3}{*}{${ }^{2} \mathbf{G L}$} & \multicolumn{6}{|c|}{ Quadrados médios } \\
\hline & & \multicolumn{3}{|c|}{ Aspergillus sp. } & \multicolumn{3}{|c|}{ Fusarium sp. } \\
\hline & & $\mathbf{C M}$ & $\operatorname{PICM}(\%)$ & IVCM & $\mathbf{C M}$ & PICM (\%) & IVCM \\
\hline Tratamento $(\mathrm{T})$ & 2 & $8487,12 *$ & $19154,39 *$ & $1077,05^{*}$ & $22201,19 *$ & $4241,84^{*}$ & $144,31^{*}$ \\
\hline Concentração (D) & 5 & $2796,95 *$ & $6313,82 *$ & $488,04^{*}$ & $5684,69 *$ & $10974,29 *$ & $776,55^{*}$ \\
\hline $\mathrm{T} * \mathrm{D}$ & 10 & $943,78^{*}$ & $2131,09^{*}$ & $83,78^{*}$ & $193,47 *$ & $372,84 *$ & $11,11 *$ \\
\hline Erro & 54 & 18,29 & 41,98 & 1,41 & 40,91 & 76,75 & 1,33 \\
\hline $\mathrm{CV}(\%)$ & & 11,31 & 15,00 & 9,59 & 20,21 & 15,62 & 12,14 \\
\hline
\end{tabular}

Por outro lado, os dados obtidos neste trabalho contradizem com os descritos por Zancan et al. (2012). Para estes, ao avaliarem a eficácia do controle químico sob o fungo Sclerotinia sclerotiorum, constataram para o grupo químico das estrobilurinas, como é o caso do fungicida Piraclostrobina, também utilizado neste trabalho, que o mesmo apresentou menor eficácia ao compará-lo com fungicidas sistêmicos. Garcia et al. (2013), ao testarem diferentes fungicidas para inibir o crescimento micelial de $S$. sclerotiorum, constataram que o fungicida azoxistrobina não foi eficiente na inibição do crescimento micelial.

Quanto ao fungo Fusarium sp., constatou-se que o fungicida Epoxiconazol + Piraclostrobina foi o que apresentou a maior porcentagem de inibição do crescimento micelial, com média superior a $70 \%$. O fungicida que promoveu a menor porcentagem foi o Fluxapiroxade + Piraclostrobina, com média inferior a 50\%, não diferindo estatisticamente do fungicida Ciproconazol + Picoxistrobina (Tabela 3).

Os dados reportados neste trabalho comprovam a eficácia de fungicidas formulados a partir da mistura de princípios ativos dos grupos químicos estrobilurinas e triazóis. Lopes et al. (2015), também relataram a eficácia de fungicidas provenientes da mistura destes grupos químicos, uma vez que, ao avaliarem a sensibilidade de isolados de Colletotrichum gloeosporioides a fungicidas in vitro, constataram que o fungicida Trifloxistrobina + Tebuconazol, promoveu menor crescimento micelial.

Quanto ao fator concentração, constatou-se que quanto maior a concentração dos fungicidas aplicados maior a porcentagem de inibição do crescimento micelial para ambos os fungos estudados (Figura 1).

Para o fungo Aspergillus sp., as concentrações de 100, 500 e 1000 ppm promoveram as maiores porcentagens de inibição do crescimento, com médias variando entre 50 e 70\%. A concentração que proporcionou a menor inibição foi a de zero ppm (Testemunha) (Figura 1).

Quanto ao fungo Fusarium sp., observou-se diferença significativa entre as diferentes concentrações utilizadas, visto que quanto maior a concentração menor o crescimento micelial. A concentração 1000 ppm, apresentou os menores crescimentos, com média pouco acima $10 \mathrm{~mm}$, não diferindo estatisticamente da concentração de 500 ppm, com valores próximos a $20 \mathrm{~mm}$ (Figura 1). 
Tabela 3. Efeito dos fungicidas sobre crescimento micelial $(\mathrm{CM})$ e a porcentagem de inibição do crescimento (PICM) de Aspergillus sp. e Fusarium sp.

\begin{tabular}{ccccc}
\hline \multirow{2}{*}{ Tratamentos (i.a) } & \multicolumn{2}{c}{ Aspergillus sp. } & \multicolumn{2}{c}{ Fusarium sp. } \\
\cline { 2 - 5 } & $\mathbf{C M}(\mathbf{m m})$ & PICM $(\%)$ & CM $(\mathbf{m m})$ & PICM $(\%)$ \\
\hline Epoxiconazol + Piraclostrobina & $28,91 \mathrm{~B}$ & $56,54 \mathrm{~B}$ & $20,68 \mathrm{~A}$ & $71,29 \mathrm{~A}$ \\
Ciproconazol + Picoxistrobina & $59,40 \mathrm{C}$ & $10,75 \mathrm{C}$ & $35,82 \mathrm{~A}$ & $50,30 \mathrm{~B}$ \\
Fluxapiroxade + Piraclostrobina & $25,08 \mathrm{~A}$ & $62,31 \mathrm{~A}$ & $38,41 \mathrm{~B}$ & $46,66 \mathrm{~B}$ \\
\hline Média & 37,80 & 43,20 & 31,64 & 56,09 \\
\hline CV (\%) & 11,31 & 15,00 & 20,21 & 15,62 \\
\hline
\end{tabular}

*Médias seguidas de mesmas letras na coluna não diferem estatisticamente entre si pelo Teste de Tukey, em nível de significância de 5\%.

Comportamento semelhante foi descrito por Lopes et al. (2015), que ao avaliarem a sensibilidade de diferentes isolados de $C$. gloeosporioides a fungicidas, observaram redução do crescimento micelial em paralelo ao aumento das concentrações dos diferentes fungicidas. Bampi et al. (2013), também observaram este comportamento em relação às concentrações e concluíram que os fungicidas do grupo químico das estrobilurinas proporcionou, mesmo em baixas concentrações, inibição do crescimento micélial de diferentes isolados de Stenocarpella macrospora.

Por outro lado, Zancan et al. (2012), ao avaliarem a eficácia do controle químico e biológico de diferentes isolados de $S$. sclerotiorum, constataram insensibilidade por parte destes isolados independente das concentrações dos fungicidas utilizadas.

Analisando o desdobramento da interação das concentrações dentro de cada tratamento (fungicida) para a porcentagem de inibição do crescimento micelial dos fungos Aspergillus sp. e
Fusarium sp., observou-se que houve diferença significativa $(p \leq 0,05)$ entre as concentrações para todos os fungicidas testados para ambos os fungos. De modo geral, quanto maior a concentração maior a porcentagem de inibição do crescimento para os diferentes fungicidas (Figura 2).

Para os resultados do fungicida Epoxiconazol + Piraclostrobina quanto ao fungo Aspergillus sp. constatou-se que as concentrações de 500 e 1000 ppm foram as que apresentaram as maiores porcentagens de inibição do crescimento micelial, com porcentagens superiores a $90 \%$. A menor porcentagem de inibição foi obtida na concentração de zero ppm (testemunha). Já quanto ao fungo Fusarium sp., observou-se que a concentração que apresentou a maior porcentagem de inibição do crescimento foi a de 1000 ppm, com média superior a 95\%, não diferindo das concentrações de 500, 100 e $50 \mathrm{ppm}$, e com valores médios variando entre 80 e $95 \%$. A menor porcentagem de inibição foi obtida na concentração de zero ppm (Testemunha) (Figura 2A). 


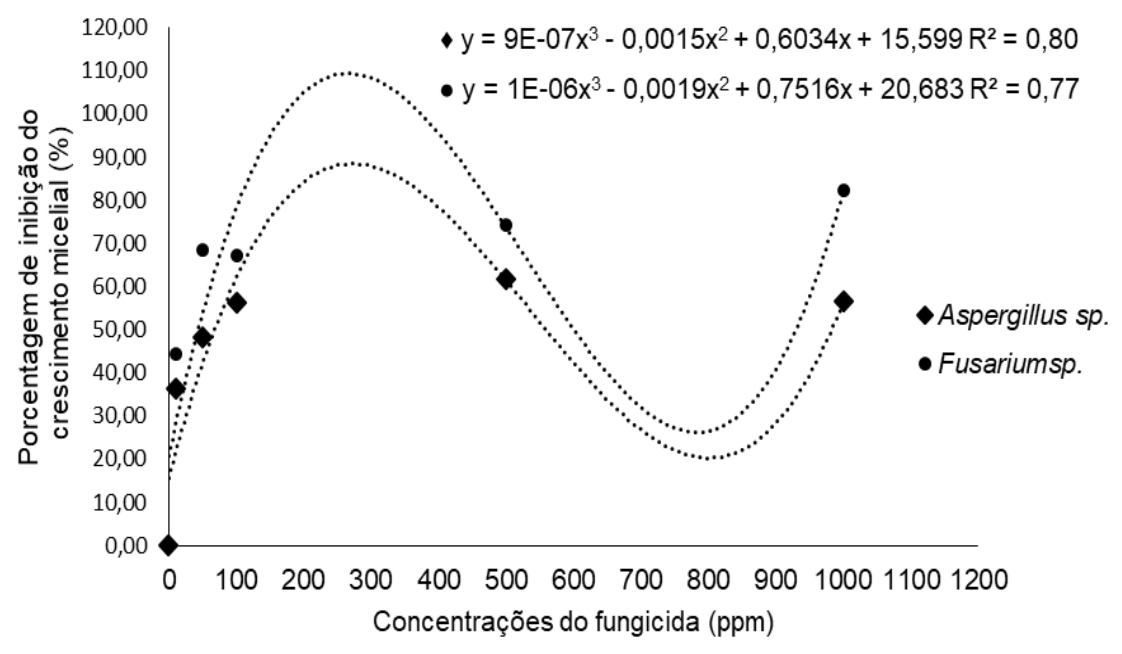

Figura 1. Porcentagem de inibição do crescimento micelial (PICM - \%) dos fungos Aspergillus sp. e Fusarium sp. sob diferentes concentrações de fungicidas.

Quanto ao fungicida Ciproconazol + Picoxistrobina para o fungo Aspergillus sp., as maiores porcentagens de inibição do crescimento micelial foram observadas nas concentrações de 100 , 500, 10, 50 e 1000 ppm, as quais não diferiram entre si, com médias variando entre 5 e $20 \%$. A concentração de zero ppm (testemunha) foi a que apresentou a menor porcentagem de inibição. Já quanto ao fungo Fusarium sp., constatou-se que a concentração de 1000 ppm proporcionou a maior porcentagem de inibição do crescimento, com média superior a $80 \%$, não diferindo estatisticamente da concentração de 500 ppm. A concentração de zero ppm (testemunha) foi a que obteve a menor porcentagem de inibição (Figura 2B).

Para $\mathrm{O}$ fungicida Fluxapiroxade + Piraclostrobina, quanto ao fungo Aspergillus sp., constatou-se que as concentrações que apresentaram as maiores porcentagens de inibição do crescimento micelial foram as de 10, 50, 100, 500 e 1000 ppm, as quais não diferiram entre si, com médias variando entre 70 e $80 \%$. A menor porcentagem de inibição foi observada na concentração de zero ppm (testemunha). Já para o fungo Fusarium sp., observou-se que a concentração que proporcionou a maior porcentagem de inibição do crescimento foi a de 1000 ppm, com média superior a 60\%, não diferindo estatisticamente das concentrações de 500, 100 e 50 ppm. A menor porcentagem de inibição foi obtida na concentração de zero ppm (Figura 2C).

Bampi et al. (2013) e Chaves Neto et al. (2016), avaliando a sensibilidade de fungos a fungicidas, também observaram diferenças entre as concentrações e descreveram que quanto maior a concentração menor o crescimento micelial, sendo consequentemente maior o efeito inibitório dos fungicidas. Além disso, estes pesquisadores constataram que os fungicidas do grupo das estrobilurinas, mesmo em baixas concentrações, proporcionaram considerável redução no crescimento micelial dos fungos estudados.

Rampersad e Teelucksingh (2012), também relataram a eficácia das estrobilurinas, ao avaliarem sensibilidade do fungo Colletotrichum spp. a diferentes fungicidas. Estes constataram que a piraclostrobina causou redução significativa no crescimento micelial.

Ao avaliar o efeito dos fungicidas e das concentrações sobre o índice de velocidade média de crescimento micelial (IVCM) do fungo Aspergillus sp. e Fusarium sp. os mesmos sofreram influência de todos os fatores estudados, bem como como pela interação entre eles, concentração $\mathrm{x}$ tratamento (fungicida) (Tabela 4). 

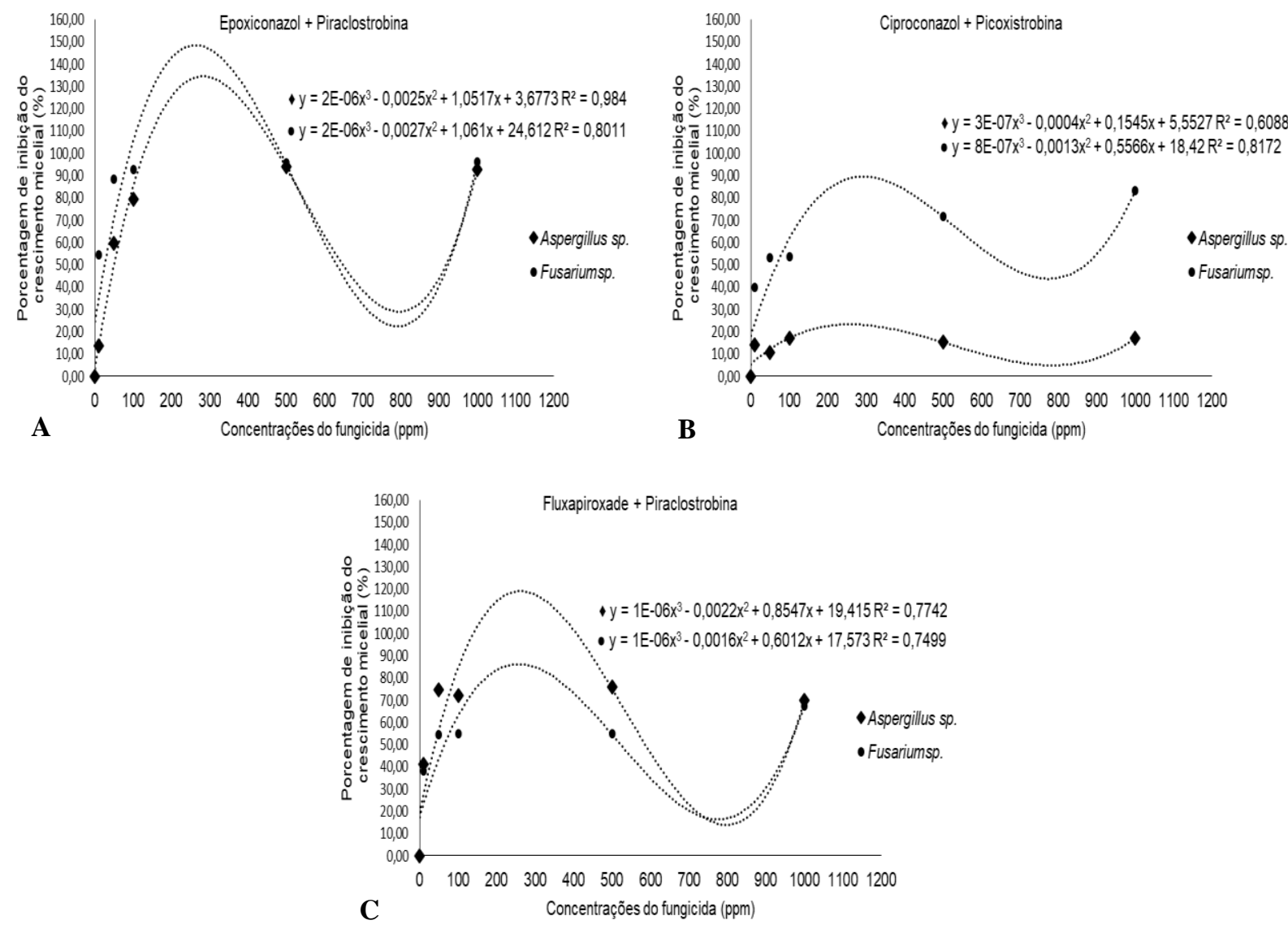

Figura 2. Desdobramento da interação concentração x fungicida. Epoxiconazol + Piraclostrobina (A), Ciproconazol + Picoxistrobina (B) e Fluxapiroxade + Piraclostrobina (C), referente a porcentagem de inibição do crescimento micelial dos fungos Aspergillus sp. e Fusarium sp.

Para o fator tratamento, quanto ao fungo Aspergillus sp., verificou-se que o fungicida Fluxapiroxade + Piraclostrobina apresentou o menor índice de crescimento, com média de $7,77 \mathrm{~mm} \mathrm{dia}^{-1}$; portanto, um menor crescimento micelial e um maior efeito inibitório ao crescimento. O maior índice de crescimento foi constatado no fungicida Ciproconazol + Picoxistrobina, com média de 20,06 $\mathrm{mm}$ dia $^{-1}$. Já quanto ao fungo Fusarium sp., constatou-se que o fungicida Epoxiconazol + Piraclostrobina foi o que apresentou o menor índice de crescimento micelial com média de $6,69 \mathrm{~mm}^{\mathrm{m}} \mathrm{dia}^{-}$ 1. O maior índice foi obtido pelo fungicida Fluxapiroxade + Piraclostrobina, com média de $11,14 \mathrm{~mm} \mathrm{dia}^{-1}$, e que este não diferiu estatisticamente do fungicida Ciproconazol + Picoxistrobina (Tabela 4).

Para o fator concentração, constatou-se, de modo geral, que houve redução significativa do crescimento micelial com o aumento da concentração para todos os fungicidas testados (Tabela 4). Quanto ao fungo Aspergillus sp. a concentração que proporcionou o menor índice de crescimento foi a de 1000 ppm, com média de 7,74 $\mathrm{mm} \mathrm{dia}{ }^{-1}$, sendo que este não diferiu estatisticamente da concentração de 500 ppm. O maior índice de crescimento foi apresentado na concentração de zero ppm, com média de 20,06 $\mathrm{mm} \mathrm{dia}^{-1}$. Já para o fungo Fusarium sp. a concentração que promoveu o menor índice de crescimento micelial foi a de 1000 ppm, 
com média de $3,57 \mathrm{~mm}$ dia $^{-1}$, diferindo estatisticamente da testemunha (concentração de zero ppm) que apresentou o maior índice em relação as diferentes concentrações (Tabela 4).

Estes resultados diferem dos obtidos por Maia et al. (2011), que ao avaliarem o efeito da temperatura no crescimento micelial, produção e germinação de conídios de Colletotrichum spp., observaram que não houve interação significativa para o índice de crescimento micelial (IVCM) em relação aos isolados analisados e a temperatura.

Quanto ao desdobramento das concentrações dentro de cada tratamento para índice de velocidade média de crescimento micelial dos fungos Aspergillus sp. e Fusarium sp., constatou-se que houve diferença significativa $(\mathrm{p} \leq 0,05)$ entre as concentrações para todos os fungicidas testados no experimento (Tabela 4).

Quanto ao fungicida Epoxiconazol + Piraclostrobina, observou-se, para o fungo Aspergillus sp., que as concentrações de 500 e 1000 ppm foram as que apresentaram os menores índices de velocidade de crescimento micelial, com média de 2,06 e 2,25 $\mathrm{mm} \mathrm{dia}^{-1}$, respectivamente; porém não diferiram da concentração de 100 ppm. O maior índice de crescimento foi obtido na concentração de zero ppm (Testemunha), com média de 24,76 mm dia $^{-1}$. Para o fungo Fusarium sp. a concentração de 1000 ppm proporcionou o menor índice de crescimento micelial, com média de 1,26 mm dia ${ }^{-1}$, não diferindo estatisticamente das concentrações de 500,100 e 50 ppm, e com média variando entre 1,55 e 2,30 $\mathrm{mm} \mathrm{dia}^{-1}$ (Tabela 4).

Para os resultados do fungicida Ciproconazol + Picoxistrobina, relacionados ao fungo Aspergillus sp., a concentração que apresentou o menor índice de velocidade de crescimento micelial foi a de 1000 ppm, com média de $16,09 \mathrm{~mm} \mathrm{dia}^{-1}$. O maior índice foi obtido na concentração de zero ppm (testemunha), com média de 24,76 mm dia ${ }^{-1}$. Já para o fungo Fusarium sp. a concentração de 1000 ppm, foi a que apresentou o menor índice de crescimento micelial, com média de $3,32 \mathrm{~mm} \mathrm{dia}{ }^{-1}$, diferindo estatisticamente da testemunha (concentração de zero ppm) que apresentou o maior índice em relação as diferentes concentrações (Tabela 4).

No fungicida Fluxapiroxade + Piraclostrobina, para o fungo Aspergillus sp., todas as concentrações foram semelhantes entre si e promoveram os menores índice de velocidade de crescimento micelial, diferindo estatisticamente da testemunha que apresentou o maior índice de crescimento micelial. Já quanto ao fungo Fusarium sp., a concentração de 1000 ppm, foi a que apresentou o menor índice de crescimento micelial, com média de $6,14 \mathrm{~mm} \mathrm{dia}{ }^{-1}$, não diferindo estatisticamente das concentrações de 500, 100 e 50 ppm, com média variando entre 7,86 e $8,39 \mathrm{~mm} \mathrm{dia}^{-1}$ (Tabela 4).

Segundo Tonin et al. (2013), monitorar a sensibilidade de determinado fungo a uma molécula química (fungicida) em especifico, é de fundamental importância para maximizar a eficiência da aplicação e a eficácia das moléculas químicas no controle de determinado patógeno. Reis et al. (2010) enfatizam que $\mathrm{IC}_{50}$ é um parâmetro específico e constante para um patógeno em particular e uma molécula química específica. Quando a substância química apresenta ação fungicida em baixa concentração, com um baixo valor $\mathrm{IC}_{50}$, esta é considerada um fungicida com elevada fungitoxicidade.

Os valores de $\mathrm{IC}_{50}$ estão apresentados na Tabela 5. Os coeficientes de determinação variaram entre 0,61 e 0,98 \% para os dois fungos avaliados. Para o fungo Aspergillus sp., os fungicidas Epoxiconazol + Piraclostrobina e Fluxapiroxade + Piraclostrobina mostraram maior inibição do crescimento micelial com concentração inibitória de $50 \%\left(\mathrm{IC}_{50}\right)$ de 49,68 e 39,78 ppm, respectivamente, sendo este fungicidas considerados moderamente fungitóxicos de acordo com a classificação de Edgington et al. (1971). O fungicida Ciproconazol + Picoxistrobina, apresentou $\mathrm{IC}_{50}$ superior a 40,00 ppm, indicando a insensibilidade por parte do fungo Aspergillus sp. 
Tabela 4. Índice de velocidade média de crescimento micelial (IVCM - mm dia ${ }^{-1}$ ) dos fungos Aspergillus sp. e Fusarium sp.

\begin{tabular}{ccccc}
\hline \multirow{2}{*}{$\begin{array}{c}\text { Concentrações } \\
(\mathbf{p p m})\end{array}$} & $\begin{array}{c}\text { Epoxiconazol }+ \\
\text { Piraclostrobina }\end{array}$ & Ciproconazol + Picoxistrobina & $\begin{array}{c}\text { Fluxapiroxade }+ \\
\text { Piraclostrobina }\end{array}$ & Média Geral \\
\cline { 2 - 4 } & \multicolumn{4}{c}{ Aspergillus sp. } \\
\hline 0 & $24,76 \mathrm{~d}$ & $24,76 \mathrm{c}$ & $24,76 \mathrm{e}$ \\
10 & $16,57 \mathrm{c}$ & $20,30 \mathrm{~b}$ & $3,50 \mathrm{a}$ & $13,46 \mathrm{~d}$ \\
50 & $6,26 \mathrm{~b}$ & $19,72 \mathrm{~b}$ & $4,54 \mathrm{a}$ & $10,17 \mathrm{c}$ \\
100 & $3,80 \mathrm{ab}$ & $20,07 \mathrm{~b}$ & $4,39 \mathrm{a}$ & $9,42 \mathrm{bc}$ \\
500 & $2,06 \mathrm{a}$ & $19,40 \mathrm{~b}$ & $4,56 \mathrm{a}$ & $8,68 \mathrm{ab}$ \\
1000 & $2,25 \mathrm{a}$ & $16,09 \mathrm{a}$ & $4,89 \mathrm{a}$ & $7,74 \mathrm{a}$ \\
\hline Média Geral & $9,29 \mathrm{~B}$ & $20,06 \mathrm{C}$ & $7,77 \mathrm{~A}$ & \\
\hline & & & $25,16 \mathrm{c}$ & $10,88 \mathrm{c}$ \\
\hline 0 & $25,16 \mathrm{c}$ & $11,26 \mathrm{~b}$ & $6,31 \mathrm{~b}$ \\
10 & $8,30 \mathrm{~b}$ & $25,16 \mathrm{e}$ & $7,86 \mathrm{a}$ & $5,89 \mathrm{~b}$ \\
50 & $2,30 \mathrm{a}$ & $13,10 \mathrm{~d}$ & $8,06 \mathrm{a}$ & $5,25 \mathrm{~b}$ \\
100 & $1,57 \mathrm{a}$ & $8,77 \mathrm{c}$ & $8,39 \mathrm{a}$ & $3,57 \mathrm{a}$ \\
\hline
\end{tabular}

\section{Média Geral}

$6,69 \mathrm{~A}$

$10,70 \mathrm{~B}$

$11,14 \mathrm{~B}$

*Médias seguidas de mesmas letras, minúsculas na coluna e maiúsculas na linha, não diferem estatisticamente entre si pelo Teste de Tukey, em nível de significância de $5 \%$.

Valores próximos aos observados neste trabalho foram descritos por Tavares e Souza (2005). Estes, ao avaliarem o efeito de fungicidas no controle in vitro de $C$. gloeosporioides, concluíram que o ingrediente ativo thiabendazol, foi classificado como moderadamente fungitóxico, com $\mathrm{IC}_{50}$ igual a $33,30 \mathrm{ppm}$, sendo este fungicida sistêmico igual aos avaliados neste trabalho. Para $\mathrm{o}$ fungicida propiconazol, pertencente ao grupo químico dos triazóis, Bolaños et al. (2012), encontraram isolados de Mycosphaerella fijiensi resistentes a esse fungicida, com valores de $\mathrm{IC}_{50}$ superiores a 40,00 ppm, sendo considerado não toxico para este fungo.

De acordo com Frac (2014), a IC $_{50}$ pode ter seu valor alterado ao longo do tempo de uso, visto que é um parâmetro específico para uma determinada substância química e microrganismo alvo. Uma sustância química é tida como fungitóxica quando apresenta um baixo valor de
$\mathrm{IC}_{50}$, o que significa alta eficácia do fungicida (Bampi et al., 2013; Santos et al., 2017).

O fungo Fusarium sp. foi considerado moderadamente sensível ao fungicida Epoxiconazol + Piraclostrobina (Tabela 5), pois que apresentou concentração inibitória de $50 \%$ ( $\mathrm{IC}_{50}$ ) de 25,56 ppm e coeficiente de determinação de $0,80 \%$, sendo classificado quanto ao nível de toxicidade como moderadamente fungitóxico (Edgington et al., 1971). Já os fungicidas Ciproconazol + Picoxistrobina e Fluxapiroxade + Piraclostrobina mostraram valores de $\mathrm{IC}_{50}$ superiores a $40,00 \mathrm{ppm}$, indicando a insensibilidade do fungo a estes fungicidas, sendo classificados como não tóxicos de acordo com Edgington et al. (1971).

Bampi et al. (2013), ao avaliarem a sensibilidade do fungo S.macrospora a fungicidas e estimarem a $\mathrm{IC}_{50}$, constataram que os fungicidas inibidores da respiração mitocondrial 
(estrobilurinas) mostraram-se altamente fungitóxicos, com $\mathrm{IC}_{50}$ para azoxistrobina de 0,16 ppm, ingrediente ativo também utilizado neste trabalho, porém em mistura com outras substâncias do grupo dos triazóis. Tonin et al. (2013), avaliando a sensibilidade micelial in vitro, medida pela $\mathrm{IC}_{50}$ de
Macrophomina phaseolina a diferentes fungicidas, descreveram o ingrediente ativo carbendazim como altamente fungitóxico com $\mathrm{IC}_{50}$ de $0,23 \mathrm{ppm}$, e como não tóxicos o fluquinconazole, com $\mathrm{IC}_{50}$ superior a $40 \mathrm{ppm}$.

Tabela 5. Fungicida, equação de regressão, coeficiente de determinação $\left(\mathrm{R}^{2}\right)$ e concentração inibitória de 50 $\%\left(\mathrm{IC}_{50}\right)$ do crescimento micelial dos fungos Aspergillus sp. e Fusarium sp.

\begin{tabular}{|c|c|c|c|c|}
\hline \multirow[t]{2}{*}{ Fungo } & \multirow[t]{2}{*}{ Equação de regressão* } & $\mathbf{R}^{2}$ & $\mathrm{IC}_{50} * *$ & \multirow[t]{2}{*}{$\mathbf{S}^{(\mathbf{1})}$} \\
\hline & & $(\%)$ & (ppm) & \\
\hline \multicolumn{5}{|c|}{ Epoxiconazol + Piraclostrobina } \\
\hline Aspergillus sp. & $y=2 E-06 x^{3}-0,0025 x^{2}+1,0517 x+3,6773$ & 0,98 & 49,68 & NT \\
\hline Fusarium sp. & $y=2 E-06 x^{3}-0,0027 x^{2}+1,061 x+24,612$ & 0,80 & 25,56 & MF \\
\hline \multicolumn{5}{|c|}{ Ciproconazol + Picoxistrobina } \\
\hline Aspergillus sp. & $y=3 E-07 x^{3}-0,0004 x^{2}+0,1545 x+5,5527$ & 0,61 & 956,97 & NT \\
\hline Fusarium sp. & $y=8 E-07 x^{3}-0,0013 x^{2}+0,5566+18,42$ & 0,82 & 66,70 & NT \\
\hline \multicolumn{5}{|c|}{ Fluxapiroxade + Piraclostrobina } \\
\hline Aspergillus sp. & $y=1 E-06 x^{3}-0,0022 x^{2}+0,8547 x+19,415$ & 0,77 & 39,78 & MF \\
\hline Fusarium sp. & $y=1 E-06 x^{3}-0,0016 x^{2}+0,6012 x+17,573$ & 0,75 & 64,59 & NT \\
\hline
\end{tabular}

De acordo com Zhang et al. (2015), os fungicidas amplamente utilizados no meio agrícola, são os do grupo químico dos triazóis que apresentam eficácia no manejo de inúmeros patógenos devido seu grau de especificidade na célula fúngica e a indução de resistência no hospedeiro, devido a maioria de seus compostos apresentarem ação sistêmica acropetal. A eficiência destes fungicidas é elevada quando se utilizou em conjunto com princípios ativos do grupo das estrobilurinas.

Os fungicidas propiconazole e azoxystrobina são utilizados há mais de 20 anos por países vizinhos ao Brasil. Porém, nos últimos anos, tem sido registrada com certa frequência a resistência de algumas espécies de fungos as substâncias químicas pertencentes aos grupos das estrobilurinas e triazóis, mesmo que sua aplicação seja feita de maneira misturada ou isoladamente (Hanada et al., 2015). Gomes et al. (2014), com base nos resultados obtidos, concluíram que houve insensibilidade por parte do isolado a alguns fungicidas. É importante salientar que o efeito fungitóxico de determinada substância química é específico entre esta substância e o microrganismo alvo, visto que uma substância em especifico não controla todos os fungos (Reis et al., 2010).

\section{CONCLUSÕES}

A porcentagem de inibição do crescimento micelial dos fungos Aspergillus sp. e Fusarium sp., de modo geral, é proporcional às concentrações dos fungicidas utilizados. Isso significa que, quanto 
maior a concentração do fungicida, maior o efeito inibitório do crescimento micelial do fungo.

Para o fungo Aspergillus sp., o fungicida Fluxapiroxade + Piraclostrobina foi eficaz por proporcionar os menores crescimentos miceliais, com $\mathrm{IC}_{50}$ de 39,78 ppm, sendo classificado como moderadamente fungitóxico.

Para o fungo Fusarium sp. o fungicida Epoxiconazol + Piraclostrobina foi eficaz por proporcionar os menores crescimentos miceliais, com $\mathrm{IC}_{50}$ de 25,56 ppm, sendo classificado como moderadamente fungitóxico.

De modo geral, ocorreu redução do índice de velocidade média de crescimento micelial dos fungos Aspergillus sp. e Fusarium sp. em paralelo ao aumento das concentrações dos fungicidas.

\section{AGRADECIMENTOS}

Os autores agradecem a Coordenação de Aperfeiçoamento de Pessoal de Nível Superior (CAPES), pelo auxílio financeiro e pela bolsa de mestrado ao primeiro autor.

\section{REFERÊNCIAS BIBLIOGRÁFICAS}

Bampi, D.; Casa, R.T.; Wordell Filho, J.A.; Blum, M.M.C.; Camargo, M.P. (2013). Sensibilidade de Stenocarpella macrospora a fungicidas. Bioscience Journal 29 (4): 787-795.

Bolaños, L.M.; Ortiz, D.T.; Maciel, J.C.R.; Aguilera, J.Á.M.; Ángel, D.N.; Flores, J.I.C.; Sánchez, D.M.; Diaz, C.N.; Aguayo, G.S. (2012). Resistência a fungicidas en poblaciones de Mycosphaerella fijiensis del sureste mexicano. Agrociencia 46(7): 707-712.

Bolton, M.D.; Rivera-Varas, V.; Del-Rio-Mendoza, L.E.; Khan, M.F.; Secor, G.A. (2012). Efficacy of variable tetraconazole rates against Cercospora beticola isolates with differing in vitro sensitivities to DMI fungicides. Plant Disease 96(12): 17491756. http://dx.doi.org/10.1094/PDIS-03-12-0255RE

Chaves Neto, J.R.; Costa, I.F.D.; Boscaini, R.; Ledur, N.R.; Guerra, R.C. (2016). Sensibilidade in vitro de fungos isolados de grãos de milho ao fungicida azoxistrobina + benzovindiflupir em diferentes concentrações. Enciclopédia Biosfera 13(23):

1211-1224. http://dx.doi.org/10.18677/Enciclopedia_Biosfera_2 016_106

Chaves Neto, J.R.; Travessini, M.; Boscaini, R.; Ledur, N.R.; Costa, I.F.D. (2017). Eficácia da aplicação foliar de fungicidas no controle de mancha-branca do milho. Revista Tecnologia \& Ciência Agropecuária 11(1): 31-36.

Edgington, L.V.; Khew, K.L.; Barron, G.L. (1971). Fungitoxic spectrum of benzimidazole compounds. $\begin{array}{llll}\text { Phytopathology } & 61(1): & 42-\end{array}$ http://dx.doi.org/10.1094/Phyto-61-42

Ferreira, D.F. (2014). Sisvar: A Guide for its Bootstrap procedures in multiple comparisons. Ciência \& Agrotecnologia 38(2): 109-112. http://dx.doi.org/10.1590/S141370542014000200001

FRAC - Fungicide Resistance Action Committee. FRAC code list (C) 2014: fungicides sorted by mode of action (including FRAC code numbering), 2014. Disponível em: http://www.frac.info/publication/anhang/FRAC_Cod e_List_2014_web.pdf. Acesso em: 01 de Jul. de 2017.

Garcia, R.A.; Juliatti, F.C. (2012). Avaliação da resistência da soja a Sclerotinia sclerotiorum em diferentes estádios fenológicos e períodos de exposição ao inóculo. Tropical Plant Pathology 37(3): 196-203. http://dx.doi.org/10.1590/S198256762012000300006

Garcia, R.A.; Juliatti, F.C.; Barbosa, K.A.G. (2013). Efeito de fungicidas e herbicidas no controle de Sclerotinia sclerotiorum. Bioscience Journal 29(6): 1989-1996.

Ghini, R.; Kimati, H. Resistência de fungos a fungicidas. Jaguariuna: Embrapa Meio Ambiente, 2009, 78 p.

Gomes, L.I.S.; Bibiano, L.B.J.; Silva, G.F.; Hanada, R.E.; Mizubuti, E.S.G. (2014). Baseline sensitivity of Brazilian Mycosphaerella fijiensis isolates to protectant and systemic fungicides. Tropical Plant Pathology 39(2):

172-177. 
http://dx.doi.org/10.1590/S1982-

56762014000200008

Hanada, R.; Gasparotto, L.; Moreira, A. (2015). Avaliação da sensibilidade de Mycosphaerella fijiensis oriundos de plátanos aos fungicidas propiconazole e azoxystrobina. Revista Ciência

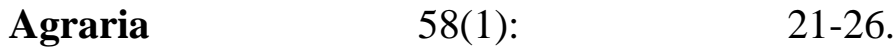
http://dx.doi.org/10.4322/rca.1697

Hawkins, N.J.; Bass, C.; Dixon, A.; Neve, P. (2018). The evolutionary origins of pesticide resistance. Biological Reviews 94(1): 135-155. https://doi.org/10.1111/brv.12440

Lopes, L.N.S.; Silva, A.S.; Pereira, C.C.O.; Menezes, I.P.P.; Malafaia, G.; Lima, M.L.P. (2015). Sensibilidade de isolados de Colletotrichum gloeosporioides a fungicidas. Multi-Science Journal 1(1): 106-114.

Maia, F.G.M.; Armesto, C.; Zancan, W.L.A.; Maia, J.B.; Abreu, M.S. (2011). Efeito da temperatura no crescimento micelial, produção e germinação de conídios de Colletotrichum spp. isolados de mangueira com sintomas de antracnose. Bioscience Journal 27(2): 205-210.

Mendes, M.C.; Von Pinho, R.G.; Machado, J.C.; Albuquerque, C.J.B.; Falquete, J.C.F. (2011). Qualidade sanitária de grãos de milho com e sem inoculação a campo dos fungos causadores de podridões de espigaspiga. Ciência e Agrotecnologia, 35(5), 931-939. https://doi.org/10.1590/S1413-70542011000500010

Oliveira, E.M.; Tanure, C.B.G.S.; Castejon, F.V.; Castro, R.M.A.D.; Rocha, F.R.T.; Carvalho, F.B.; Andrade, M.A; Stringhini, J.H. (2015). Performance and nutrient metabolizability in broilers fed diets containing corn contaminated with Fumonisin B1 and esterified glucomannan. Revista Brasileira de Ciência Avícola, Campinas 17(3): 313-318.

Pfeufer, E.E.; Ngugi, H.K. (2012). Orchard factors associated with resistance and cross resistance to sterol demethylation inhibitor fungicides in populations of Venturia inaequalis from Pennsylvania. Disease Control and Pest Management 102(3): 272-282. http://dx.doi.org/10.1094/PHYTO-04-11-0117
Rampersad, S.N.; Teelucksingh, L.D. (2012). Differential responses of Colletotrichum gloeosporioides and $C$. truncatum isolates from different hosts to multiple fungicides based on two assays. Plant Disease 96(10): 1526-1536. https://doi.org/10.1094/PDIS-10-11-0906-RE

Reis, E.M.; Reis, A.C.; Carmona, M.A. Manual de fungicidas: Guia para controle químico de doenças de plantas. 6. ed. Passo Fundo: UPF, 2010. $226 \mathrm{p}$.

Rufatto, M. 2014. Micotoxinas e acometimentos à saúde humana - ênfase no potencial carcinogênico. Revista Brasileira de Nutrição Clínica Funcional ano 14 , no 60.

Santos, L.A.; Marek, J.; Werner, L.C.; Mariano, L.C.; Faria, C.M.D.R. (2017). Sensibilidade micelial in vitro de Cercospora beticola ao oxicloreto de cobre. Scientia Agraria 16(2): 153-157. http://dx.doi.org/10.18188/1983-

1471/sap.v16n1p153-157

Silva, I.F.; Martins, P.R.; Silva, E.G.; Ferreira, S.B.; Ferreira, V.F.; Costa, K.R.; Vasconcellos, M.C.; Lima, E.S.; Silva, F.C. (2013). Synthesis of 1H1,2,3-triazoles and study of their antifungal and cytotoxicity activities. Medicinal Chemistry 9(8): 1085-1090.

http://dx.doi.org/10.2174/1573406411309080010

Souza, A.G.C.; Maffia, L.A.; Mizubuti, E.S.G. (2012). Cultural and aggressiveness variability of Cercospora coffeicola. Journal of Phytopathology 160(10): 540-546. https://doi.org/10.1111/j.14390434.2012.01947.x

Tavares, G.M.; Souza, P.E. (2005). Efeito de fungicidas no controle in vitro de Colletotrichum gloeosporioides, agente etiológico da antracnose do mamoeiro (Carica papaya L.). Ciência e $\begin{array}{lll}\text { Agrotecnologia } & 29(1): & 52-59 .\end{array}$ http://dx.doi.org/10.1590/S141370542005000100006

Tonin, R.F.B.; Avozani, A.; Danelli, A.L.D.; Reis, E.M.; Zoldan, S.M.; Garcés-Fiallos, F.R. (2013). In vitro mycelial sensitivity of Macrophomina phaseolina to fungicides. Pesquisa Agropecuária Tropical 43(4): 460-466. 
http://dx.doi.org/10.1590/S1983-

40632013000400014

Vitorino, O.C.L. 2011. Micotoxinas na Alimentação e na Saúde Animal e Humana. Dissertação (Mestrado em Engenharia Zootécnica) Departamento de Ciências Agrárias, UAC, Universidade dos Açores, Angra do Heroísmo.

Zancan, W.L.A.; Machado, J.C.; Sousa, B.F.M.; Matos, C.S.M. (2012). Crescimento micelial, produção e germinação de Escleródios de Sclerotinia sclerotiorum na presença de fungicidas químicos e Trichoderma harzianum. Bioscience Journal 28(5): 782-789.

Zhang, Y.; Smith, P.; Maximova, S.N.; Guiltinan, M.J. (2015). Applying Glycerol as a Foliar Spray Activates the Defense Response and Enhances Disease Resistance of Theobroma cacao. Molecular plant pathology 16(16): 27-37. https://doi.org/10.1111/mpp.12158

Zhou, C.H.; Wang, Y. (2012). Recent Researches in Triazole Compounds as Medicinal Drugs. Current Medicinal Chemistry 19(2): 239-280. https://doi.org/10.2174/092986712803414213 